\title{
Screening for Coeliac Disease: Are We Good at It?
}

Montasser Nadeem"

\begin{abstract}
Department of Paediatrics, Our Lady's Chi
Coeliac disease (CD) is an immune reaction to gluten in genetically predisposed subjects. CD is likely to affect approximately 1\% of the population of England [1]. The age of presentation is influenced by the age of introduction of gluten into the diet. CD may present as a large variety of non-specific signs and symptoms. Children with $\mathrm{CD}$ may experience unexplained poor growth following the introduction of gluten, delay puberty, abnormal stools and constipation. General irritability, chronic abdominal pain, abnormal liver biochemistry, abdominal distension and buttock wasting have been reported. CD should be considered in children presented with iron deficiency anaemia, growth failure with or without gastrointestinal symptoms. Patients with CD may experience low bone mineral density [2], but similar health-related quality of life as their peers without CD [3]. However individuals with CD may exhibit no symptoms. Certain individuals are at an increased risk; such as patients with type 1 diabetes mellitus, Down's syndrome and Turner's syndrome, for example, and presymptomatic screening is advised [4].

Diagnosis can be confirmed by flat mucosa on jejunal biopsy followed by the resolution of symptoms and catch up growth upon gluten withdrawal. Diagnostic serological tests have been developed. These's include IgA anti-tissue transglutaminase (IgA-tTG) and antiendomysium antibody (EMA) values. It is important to measure serum IgA level and IgG-tTG values should be considered in those with low IgA levels. In previous study, biopsyproven CD and positive EMA were reported in 60\% of those with elevated IgA-tTG levels above the reference range, whereas biopsy-proven $\mathrm{CD}$ was reported in all
\end{abstract}

dren's Hospital, Crumlin, Dublin, Ireland

patients with positive EMA [5]. European Society for Paediatric Gastroenterology, Hepatology and Nutrition working group suggested that typing for HLA-DQ2 and HLA-DQ8 can be helpful where there is uncertain diagnosis of $\mathrm{CD}$; such as cases with negative $\mathrm{CD}$ antibodies and mild changes on biopsy or with strong clinical suspicion, highly specific CD antibodies and biopsies are not going to be performed, for example [4]. Monitoring for $\mathrm{CD}$ is required in children with unexplained poor growth, delayed puberty or unexplained gastrointestinal symptoms and screening in asymptomatic individuals who are at an increased risk is advised.

\section{References}

[1] West J, Logan RF, Hill PG, Lloyd A, Lewis S, Hubbard R, Reader R, Holmes GK, Khaw KT. Seroprevalence, correlates, and characteristics of undetected coeliac disease in England. Gut 2003; 52:960-965.

[2] Kalayci AG, Kansu A, Girgin N, et al. Bone mineral density and importance of a gluten-free diet in patients with celiac disease in childhood. Pediatrics 2001; 108:E89.

[3] Nordyke K, Norström F, Lindholm L, et al. Health-related quality of life in adolescents with screening-detected celiac disease, before and one year after diagnosis and initiation of gluten-free diet, a prospective nested case-referent study. BMC Public Health 2013; 13:142.

[4] Jenkins HR, Murch SH, Beattie RM; Coeliac Disease Working Group of British Society of Paediatric Gastroenterology, Hepatology and Nutrition. Diagnosing coeliac disease. Arch Dis Child 2012; 97:393-394.

[5] Nadeem M, Roche EF. Coeliac disease in Turner syndrome. Arch Dis Child 2013; 98:649-650. 